\title{
High-coverage targeted lipidomics revealed dramatic lipid compositional changes in asthenozoospermic spermatozoa and inverse correlation of ganglioside GM3 with sperm motility
}

Shuqiang Chen ${ }^{\dagger}$, Ming Wang ${ }^{\dagger}$, Li Li ${ }^{\dagger}$, Jun Wang, Xuhui Ma, Hengde Zhang, Yang Cai, Bin Kang, Jianlei Huang ${ }^{*}$ and Bo Li* (D)

\begin{abstract}
Background: It has been previously demonstrated that cholesterol content and cholesterol/phospholipid ratio were significantly higher in asthenozoospermia and oligoasthenoteratozoospermia. The majority of published studies have investigated the fatty acid composition of phospholipids rather than lipids themselves. This study evaluated the lipid composition of asthenozoospermic and normozoospermic spermatozoa, and identified the exact lipid species that correlated with sperm motility.
\end{abstract}

Methods: A total of 12 infertile asthenozoospermia patients and 12 normozoospermia subjects with normal sperm motility values were tested for semen volume, sperm concentration, count, motility, vitality and morphology. Highcoverage targeted lipidomics with 25 individual lipid classes was performed to analyze the sperm lipid components and establish the exact lipid species that correlated with sperm motility.

Results: A total of 25 individual lipid classes and 479 lipid molecular species were identified and quantified. Asthenozoospermic spermatozoa showed an increase in the level of four lipid classes, including Cho, PE, LPI and GM3. A total of 48 lipid molecular species were significantly altered between normozoospermic and asthenozoospermic spermatozoa. Furthermore, the levels of total GM3 and six GM3 molecular species, which were altered in normozoospermic spermatozoa versus asthenozoospermic spermatozoa, were inversely correlated with sperm progressive and total motility.

Conclusions: Several unique lipid classes and lipid molecular species were significantly altered between asthenozoospermic and normozoospermic spermatozoa, revealing new possibilities for further mechanistic pursuits and highlighting the development needs of culture medium formulations to improve sperm motility.

Keywords: Asthenozoospermia, Lipidomics, Sperm motility, GM3

\footnotetext{
* Correspondence: 13572083125@163.com; Ibtn2000@126.com

†Shuqiang Chen, Ming Wang and Li Li contributed equally to this work. Department of Obstetrics and Gynecology, Tangdu Hospital, the Fourth Military Medical University, 710038 Xi'an, China
}

C C The Author(s). 2021 Open Access This article is licensed under a Creative Commons Attribution 4.0 International License, which permits use, sharing, adaptation, distribution and reproduction in any medium or format, as long as you give appropriate credit to the original author(s) and the source, provide a link to the Creative Commons licence, and indicate if changes were made. The images or other third party material in this article are included in the article's Creative Commons licence, unless indicated otherwise in a credit line to the material. If material is not included in the article's Creative Commons licence and your intended use is not permitted by statutory regulation or exceeds the permitted use, you will need to obtain permission directly from the copyright holder. To view a copy of this licence, visit http://creativecommons.org/licenses/by/4.0/. The Creative Commons Public Domain Dedication waiver (http://creativecommons.org/publicdomain/zero/1.0/) applies to the data made available in this article, unless otherwise stated in a credit line to the data. 


\section{Introduction}

The plasma membrane is an essential structural component of living cells, which serves as a specialized barrier separating the cell interior from the external environment and enabling intracellular organelles to be compartmentalized [1,2]. The plasma membrane lipids play vital roles in a variety of physiological and pathological processes by sustaining the structure and function of organelles [1, 3]. Plasma membrane lipids contain four main classes: glycerophospholipids, sphingolipids, glycolipids and sterols [4]. These lipids exhibit critical biological functions, including energy storage, membrane structure construction, cell signaling and transcriptional regulation [5]. Drastically altered levels of distinct lipid species were correlated with cellular processes $[2,4]$.

The fluidity and flexibility of cell membranes are mainly dependent on their lipid constitution [6]. Integrity and fluidity of sperm membranes are important for sperm motility, capacitation, acrosome reaction (AR), and sperm-egg fusion [7]. Sperm membrane components, particularly lipids, are directly involved in core sperm functions, including membrane fluidity, sperm motility and spermatozoon-oocytecross-talk $[6,8]$. The sperm plasma membrane lipids are roughly made up of $70 \%$ phospholipids, $25 \%$ neutral lipids, and $5 \%$ glycolipids [9]. The fatty acid composition of spermatozoaplasma membrane is directly related to sperm motility $[10,11]$. Cholesterol is important for modulating the membrane fluidity, and its levels in the sperm membrane of infertile patients are dramatically higher than those in fertile men [12].

Approximately $15 \%$ of couples are affected by infertility globally, and $50 \%$ of infertile couples are due to male-factor infertility [13]. Asthenospermia, defined as total sperm motility $<40 \%$ and progressive motility $<$ $32 \%$ in a semen sample, is one of the common causes of male infertility, affecting approximately $40 \%$ of all cases $[14,15]$. It was found that spermatozoal stearic acid was negatively correlated, and DHA was positively correlated with sperm motility [16].The concentration of phosphatidylcholine (PC) and phosphatidylethanolamine (PE) of sperm membrane were reduced in asthenozoospermic samples [17]. However, the sperm lipid composition of normospermic men and lipid changes in asthenozoospermic semen samples have not been fully explored. Advances in lipidomics are enabling detailed understanding of lipids in biological membranes [18-20]. Lipidomics is the study of the entire lipid pool within a cell, tissue, or organism, which provides a quantitative analysis of the lipid profile in that sample [21]. Lipidomics has already been used to elucidate the fatty acid groups or classes in spermatozoa from different species [22]. However, quantifying the levels or amounts of lipids has proven to be more difficult than detecting their presence. This is a rather difficult task when the cells number is limited and the quantification of the lipids needs to be set to a higher threshold level than the current one [23]. With technological advancements in MS, recent efforts have been made to design a streamlined method to fractionate and quantify the lipids in sperm cells via HPLC/MSbased approaches [24].

Understating the lipids related to sperm motility is necessary to help the researchers to address the subject of reduced sperm motility and produce an accurate diagnosis of infertility [6]. The purpose of this study was to investigate the lipid composition of male sperm in normozoospermic spermatozoa and asthenozoospermic semen samples in order to better elucidate the role of lipids on male fertility. In the precent study, Exion UPLC coupled with a SCIEX QTRAP 6500 PLUS system was first used for the study of lipidomics in human spermatozoa. The targeted library tailored for human sperm lipidome conferred sufficient lipid coverage to render global lipid pathway analysis [25].

\section{Materials and methods \\ Ethical approval}

This study was approved by the Internal Review and Ethics Boards of Tangdu Hospital, and written informed consent was obtained from all participants at enrollment. This study was conducted in accordance with the Declaration of Helsinki [26].

\section{Study population}

The present study was performed at the Reproductive Unit of the Department of Obstetrics, Tangdu Hospital of the Fourth Military Medical University from January 2018 to August 2019. All participants had no diagnosed diseases related to mitochondrial dysfunction as well as no history of smoking and alcohol intake. Semen samples were collected from 12 healthy men with normal sperm concentration, total sperm count, and total and progressive mobility, as well as 12 asthenozoospermia patients who masturbated after three days of abstinence in the course of infertility work-up. We excluded participants with azoospermia, chromosome alterations, anti-sperm antibodies, undescended testis, varicocele, history of epididymitis, orchitis, epididymo-orchitis, human papillomavirus infection and/or sexually transmitted infections because of their proved negative influence on fertility and semen parameters according to previous reports [27-29]. The protocol was approved by the Internal Review and Ethics Boards of Tangdu Hospital, and written informed consent was obtained from all healthy males and patients. 


\section{Sample preparation}

After liquefaction, each semen sample was analyzed according to the World Health Organization criteria (WHO, 2010). Patients were excluded upon identification of any significant androgenic or endocrine abnormalities. The patients found to have chromosomal aberrations by karyotype testing were also excluded. Samples with abnormal $\mathrm{pH}$ and viscosity, increased number of leukocytes or immature germ cells, and abnormal semen liquefaction were excluded. Asthenozoospermia was defined as the progressive motility of sperm $<32 \%$ within 60 min of ejaculation. The fresh human semen specimens were centrifuged using a twolayer gradient system including an upper layer solution of $40 \%$ Percoll gradient and a lower layer solution of $80 \%$ Percoll gradient (GE Healthcare, Waukesha, WI, USA) (400 g, $15 \mathrm{~min}$ ) to separate spermatozoa from seminal plasma. Spermatozoa were then washed twice with $2.0 \mathrm{ml} \mathrm{PBS}$ at $300 \mathrm{~g}$ for $5 \mathrm{~min}$. Each sample was diluted to $1.0 \mathrm{ml}$ with $\mathrm{PBS}$, and then the sperms count were calculated by a hemocytometer as previously described [30]. Subsequently, $100 \mu \mathrm{L}$ of sperm solution was prepared at a concentration of $1 \times 10^{8}$ cells $/ \mathrm{ml}$ for lipid extraction.

\section{Lipid extraction}

Lipids were extracted from the sperm samples using a modified version of the Bligh and Dyer's method as previously described [31]. Briefly, $750 \mu \mathrm{l}$ of ice-cold chloroform: methanol $(1: 2, \mathrm{v} / \mathrm{v})$ with $10 \%$ deionized $\mathrm{H}_{2} \mathrm{O}$ was added to inactivate the samples. Then samples were homogenized on an automated bead ruptor (Omni, USA) and further centrifuged at $1500 \mathrm{rpm}$ and $4{ }^{\circ} \mathrm{C}$ for $1 \mathrm{~h}$. Next, $350 \mu \mathrm{l}$ of deionized $\mathrm{H}_{2} \mathrm{O}$ and $250 \mu \mathrm{l}$ of chloroform were added to generate separate phase. After transferring the lower organic phase into another tube, $500 \mu \mathrm{l}$ of chloroform was added for a second extraction. The two extractions were pooled and dried using SpeedVac (Genevac, UK). The dried samples were stored at $-80{ }^{\circ} \mathrm{C}$ for mass spectrometric analysis.

\section{Targeted lipidomics}

\section{Analysis of polar lipids}

The detailed analytical protocol was described previously $[31,32]$. Briefly, the individual lipid class of polar lipids were separated by normal phase (NP)-HPLC using a Phenomenex Luna $3 \mu \mathrm{m}$ silica column (i.d.150 $\times 2.0$ $\mathrm{mm}$ ) under the following conditions: mobile phase A (chloroform:methanol:ammonium hydroxide, 89.5:10: 0.5 ) and mobile phase B (chloroform:methanol:ammonium hydroxide:water, 55:39:0.5:5.5), the flow rate was $270 \mu \mathrm{l} / \mathrm{min}$, and column oven temperature was $25{ }^{\circ} \mathrm{C}$. The gradient started with $5 \%$ of $\mathrm{B}$ and was held for $3 \mathrm{~min}$, then increased to $40 \%$ of B over $9 \mathrm{~min}$, and held at $40 \%$ for 4 min before further increasing to $70 \%$ of B over $5 \mathrm{~min}$. The gradient was maintained at $70 \%$ of $\mathrm{B}$ for $15 \mathrm{~min}$ before returning to $5 \%$ of B over $3 \mathrm{~min}$, and was finally equilibrated for $6 \mathrm{~min}$. Individual polar lipid species were quantified by referencing the spiked internal standards including PC-14:0/14:0, d31-PC16:0/18: 1, d31-PE-16:0/18:1, d31-PS-16:0/18:1, PS-17:0/20:4, PA17:0/17:0, PA-17:0/20:4, PG-14:0/14:0, d31-PG-16:0/18: 1, GluCer-d18:1/8:0, Cer-d18:1/17:0, C14:0-LBPA, d31PI-16:0/18:1， S1P-d17:1， Sph-d17:1， SM-d18:1/12:0, LPC-17:0, LPE-17:0, LPI-17:0, LPA-17:0, LPS-17:0 obtained from Avanti Polar Lipids (Alabaster, AL, USA) and PI-8:0/8:0 from Echelon Biosciences, Inc. (Salt Lake City, UT, USA). GM3 species were quantified using GM3 d18:1/17:0 synthesized in-house as an internal standard.

\section{Analysis of neutral lipids}

Neutral lipids (TAGs, DAGs and CEs) were analyzed using a modified version of reverse-phase HPLC/MRM as previously described [33, 34]. Briefly, the lipids were separated on a Phenomenex Kinetex $2.6 \mu \mathrm{m} \mathrm{C18}$ column (i.d. $4.6 \times 100 \mathrm{~mm}^{2}$ ) using an isocratic mobile phase of chloroform: methanol:0.1 $\mathrm{M}$ ammonium acetate (100:100:4) at a flow rate of $170 \mu \mathrm{l} / \mathrm{min}$ for $17 \mathrm{~min}$. Levels of short, medium, and long-chain TAGs were calculated by referencing the spiked internal standards of TAG(14:0)3-d5, TAG(16:0)3-d5 and TAG(18:0)3-d5 obtained from CDN isotopes, respectively. DAGs were quantified using d5-DAG16:0/16:0 and d5DAG18:1/18:1 (Avanti Polar Lipids) as internal standards. Free cholesterols and cholesteryl esters were analyzed as previously described with d6cholesterol and d6-C18:0 cholesteryl ester (CE) (CDN isotopes) as internal standards.

\section{Analysis of free cholesterol}

Free cholesterols and total cholesteryl esters were analyzed using HPLC/APCI/MS/MS as previously described, with d6-Cho and d6-CE-18:1 (CDN isotopes) as internal standards [31].

\section{Statistical analyses}

The statistical differences between the normozoospermic and asthenozoospermic sample were determined using Student's $t$-test or Wilcoxon rank-sum test with Shapiro-Wilk Normality test. Correlations were assessed by Pearson's correlation coefficient (r). All analyses were performed using SPSS. Results were considered to be statistically significant if $p<0.05$. For all analyses, "*p represented $p<0.05,{ }^{* * *}$ p represented $p<0.01$. 


\section{Results}

Patients andsemen samples

A total of 12 patients with asthenozoospermia and 12 healthy fertile men were included in this study. The mean age was 33.1 years for asthenozoospermia patients, and 32.4 years for healthy fertile men. There was no significant difference between the two groups. The parameters of the semen samples were listed in Table 1. The progressive and average sperm motility of asthenozoospermia patients was $26.42 \pm 4.17 \%$ and $48.42 \pm 11.46 \%$, respectively, which were significantly lower than those of healthy fertile men $(p<0.0001$ and $p<0.0001$, respectively). There was no difference in sperm concentration, ejaculate volume and healthy sperm morphology between the two groups.

The levels of individual lipid class in normozoospermic and asthenozoospermic spermatozoa

A total of 25 lipid classes were identified and quantified in normozoospermic and asthenozoospermic spermatozoa (Fig. 1, Supplementary table-1), which included cholesterol (Cho), cholesteryl esters (CE), triacylglycerols (TAG), diacylglycerols (DAG), free fatty acids (FFA), phosphatidylcholines (PC), phosphatidylethanolamines (PE), phosphatidylinositols (PI), phosphatidylglycerols (PG), phosphatidylserines (PS), phosphatidic acids (PA), lysophosphatidylcholine (LPC), lysophosphatidylethanolamine (LPE), lysophosphatidylserine (LPS), lysophosphatidic acid (LPA), lysophosphatidylinositols (LPI), sphingomyelins (SM), ceramides (Cer), glucosylceramides (GluCer), lactosylceramides (LacCer), monosialodihexosyl gangliosides (GM3), sphingosine, (Sph), acylcarnitine, cardiolipins (CL) and ceramide trihexoside (Gb3).

The analyses of the total lipid fraction of individual lipid class were shown in Fig. 1 and Supplementary table-1. Surprisingly, contrary to normal lipid composition observed in human cells, Cho was the most abundant lipid species in normozoospermic and asthenozoospermic spermatozoa, followed by PC, SM, PE, PS, FFA, CL and TAG. Cho, PE and LPI in asthenozoospermic spermatozoa were significantly higher than those in normal controls (both $\mathrm{p}<0.05$ ) (Fig. 1). The level of GM3 in asthenozoospermic spermatozoa was significantly increased compared to normozoospermic spermatozoa, whereas plasmalogen PC, CL, PG and LPC showed a similar but insignificant trend (Fig. 1). The levels of DAG and LPS were lower in asthenozoospermic spermatozoa than those in the normal controls, but the difference was not significant (Fig. 1).

\section{Significantly altered lipid molecular species between} normozoospermic and asthenozoospermic spermatozoa A total of 479 lipid molecular species were identified and quantified in the study, of which 48 species significantly changed between normozoospermic and asthenozoospermic spermatozoa $(p<0.05) \quad$ (Fig. 2, Supplementary table-2). The altered lipid molecules belonged to PE, PC, PG, CL, LPI, LPS, Cer, GM3, DAG and TAG. PE plays a major role in the structure and function of mitochondrial membranes. In this study, the concentrations of nine PE molecules in asthenozoospermic spermatozoa were higher than those in control spermatozoa $(p<0.05)$ (Fig. 2). CL is a universal component of mitochondria in all eukaryotes, which is localized to the inner mitochondrial membrane (IMM) and plays an important role in the structural organization and the function of mitochondrial membranes [35, 36]. In this study, $55 \mathrm{CL}$ molecular species were detected, and the concentrations of $16 \mathrm{CL}$ molecules in asthenozoospermic spermatozoa were significantly increased compared to the control group $(p<0.05)$ (Fig. 2). GM3, the main ganglioside in majority of extraneural tissues of vertebrates, is one of the essential components of plasma

Table 1 The parameters of the semen samples

\begin{tabular}{lccr}
\hline Parameters studied & Normozoospermia & Asthenozoospermia & \multicolumn{1}{c}{ value } \\
\hline Age of patients & $32.417 \pm 4.716$ & $33.083 \pm 3.752$ & 0.7172 \\
Sexual abstinence (days) & $3.667 \pm 1.374$ & $4.833 \pm 1.280$ & 0.0514 \\
pH & $7.383 \pm 0.055$ & $7.400 \pm 0.000$ & 0.3283 \\
Total sperm number $(\times$ 10^6) & $560.70 \pm 278.23$ & $465.22 \pm 195.67$ & 0.3620 \\
Ejaculate volume $(\mathrm{ml})$ & $4.500 \pm 1.256$ & $3.979 \pm 1.469$ & 0.4238 \\
Sperm concentration $(\times 10 \wedge 6 / \mathrm{ml})$ & $133.000 \pm 73.389$ & $120.917 \pm 44.000$ & 0.6441 \\
Progressive motility $(\mathbf{A}+\mathbf{B})(\%)$ & $53.700 \pm 8.224$ & $26.417 \pm 4.166$ & $<0.0001 * *$ \\
Total motility & $70.750 \pm 10.762$ & $48.421 \pm 11.457$ & $0.0001 * *$ \\
Sperm vitality $(\%)$ & $84.167 \pm 5.928$ & $68.625 \pm 8.070$ & $<0.0001 * *$ \\
Good sperm morphology $(\%)$ & $6.500 \pm 2.021$ & $5.375 \pm 1.570$ & 0.1589 \\
\hline
\end{tabular}

Data are presented as Means \pm SD

Significant at ${ }^{*} P<0.05$; ${ }^{* *} P<0.01$ (by t test) 

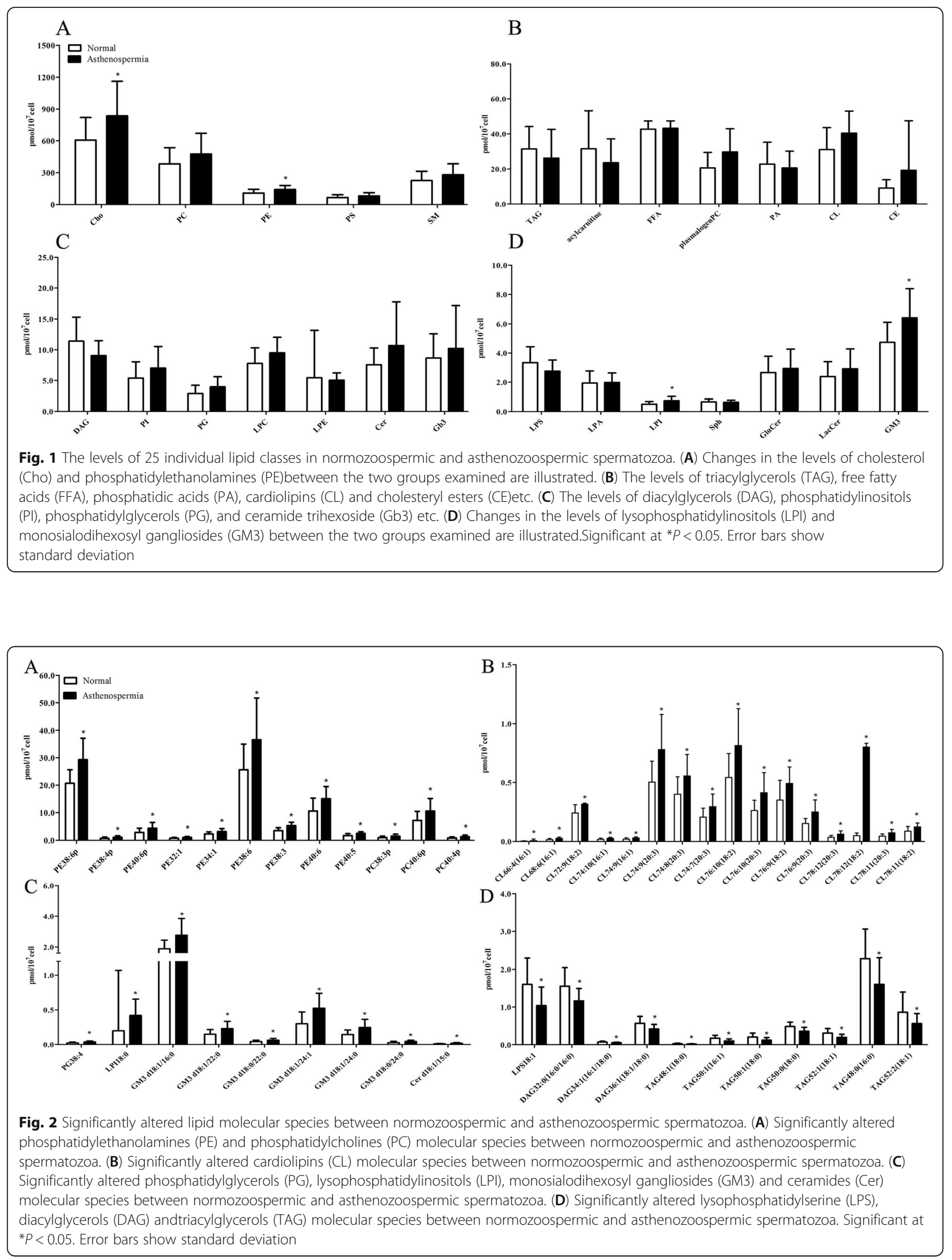
membrane rafts [37]. Twenty GM3 molecular species were identified, and the concentrations of six GM3 molecules in asthenozoospermic spermatozoa were significantly increased compared to the control group $(p<$ 0.05) (Fig. 2). TGs stored in lipid droplets (LDs) are energy substrates for $\beta$-oxidation, and precursors for membrane lipids and signaling molecules. The concentrations of seven TG molecules in asthenozoospermic spermatozoa were significantly decreased compared to the control group $(p<0.05)$ (Fig. 2).

The levels of GM3 species were inversely correlated with sperm progressive motility

Furthermore, we aimed to explore the relationship between lipids levels and sperm progressive motility. The levels of total GM3 and LPI were negatively associated with sperm progressive motility $(p<0.05)$ (Table 2$)$. The correlation of 48 significantly altered lipid species with sperm progressive motility was analyzed between normozoospermic and asthenozoospermic spermatozoa. The six significantly changed GM3 molecular species in normozoospermic spermatozoa versus asthenozoospermic spermatozoa included GM3 d18:1/16:0, GM3 d18:0/ 22:0, GM3 d18:0/24:0, GM3 d18:1/22:0, GM3 d18:1/24:0 and GM3 d18:1/24:1, which were inversely correlated with sperm progressive motility $(p<0.05)$ (Table 2$)$. The levels ofLPI18:0, PE32:1, PE34:1 and PE38:6p were also negatively correlated with sperm progressive motility $(p<0.05)$ (Table 2). Interestingly, the only lipid that was positively correlated with sperm progressive motility was TAG52:1(18:1) $(p<0.05)$ (Table 2).

Table 2 Correlation of the lipids in spermatozoa with progressive motility $(n=24)$

\begin{tabular}{lcc}
\hline Lipids & Pearson correlation coefficient & $\boldsymbol{P}$ value \\
\hline Total GM3 & -0.443 & 0.03 \\
Total LPI & -0.406 & 0.049 \\
GM3 d18:1/16:0 & -0.451 & 0.027 \\
GM3 d18:0/22:0 & -0.481 & 0.017 \\
GM3 d18:0/24:0 & -0.479 & 0.019 \\
GM3 d18:1/22:0 & -0.408 & 0.048 \\
GM3 d18:1/24:0 & -0.424 & 0.039 \\
GM3 d18:1/24:1 & -0.527 & 0.008 \\
LPI18:0 & -0.467 & 0.022 \\
PE32:1 & -0.426 & 0.038 \\
PE34:1 & -0.419 & 0.042 \\
PE38:6p & -0.416 & 0.043 \\
TAG52:1(18:1) & 0.412 & 0.045 \\
\hline Colrstions of lipids with progessive
\end{tabular}

Correlations of lipids with progressive motility were tested by Pearson's correlation analysis.Results were considered to be statistically significant if $P<0.05$
The levels of GM3 species were negatively correlated with sperm total motility

We further explored the relationship between lipids levels and sperm total motility. The concentrations of total Cho, GM3, LPI, PE and plasmalogen PC were negatively correlated with sperm total motility $(p<0.05)$ (Table 3). The correlation of 48 lipid molecular species with sperm total motility was also analyzed between normozoospermic and asthenozoospermic spermatozoa. The levels of five GM3 molecular species significantly changed between normozoospermic and asthenozoospermic spermatozoa, including GM3 d18:1/16:0, GM3 d18:0/24:0, GM3 d18:1/22:0, GM3 d18:1/24:0 and GM3 d18:1/24:1, which were negatively correlated with sperm progressive motility $(p<0.05)$ (Table 3$)$. The levels of six significantly changed PE molecular species between normozoospermic and asthenozoospermic spermatozoa included PE32:1, PE38:4p, PE38:6p, PE38:6, PE40:6p and PE40:6, which were negatively correlated with sperm progressive motility $(p<0.05)$ (Table 3$)$. The levels of LPI18:0, PC40:4p and PG38:4 were also negatively correlated with sperm progressive motility $(p<0.05)$ (Table 3).

Table 3 Correlation of the lipids in spermatozoa with total motility $(n=24)$

\begin{tabular}{lcc}
\hline Lipids & Pearson correlation coefficient & $\boldsymbol{P}$ value \\
\hline Total Cho & -0.489 & 0.015 \\
Total GM3 & -0.533 & 0.007 \\
Total LPI & -0.551 & 0.005 \\
Total PE & -0.523 & 0.009 \\
Total plasmalogenPC & -0.434 & 0.034 \\
GM3 d18:1/16:0 & -0.536 & 0.007 \\
GM3 d18:0/24:0 & -0.509 & 0.011 \\
GM3 d18:1/22:0 & -0.455 & 0.026 \\
GM3 d18:1/24:0 & -0.492 & 0.015 \\
GM3 d18:1/24:1 & -0.586 & 0.003 \\
LPI18:0 & -0.543 & 0.006 \\
PC40:4p & -0.444 & 0.030 \\
PE32:1 & -0.434 & 0.034 \\
PE38:4p & -0.524 & 0.009 \\
PE38:6p & -0.649 & 0.001 \\
PE38:6 & -0.459 & 0.024 \\
PE40:6p & -0.484 & 0.017 \\
PE40:6 & -0.493 & 0.014 \\
PG38:4 & -0.565 & 0.004 \\
\hline Cors &
\end{tabular}

Correlations of lipids with progressive motility were tested by Pearson's correlation analysis. Results were considered to be statistically significant if $\mathrm{P}<0.05$ 


\section{Discussion}

In the present study, we presented the first detailed overview of the lipid composition of human sperm from normozoospermic spermatozoa samples. A total of 25 individual lipid classes, 479 lipid molecular species were identified and quantified by high-coverage targeted lipidomics. We also evaluated the differences in lipid components between normozoospermic andasthenozoospermic spermatozoa. The results showed that lipid alterations associated with asthenospermia included increased levels of cholesterol (Cho), PEs, LPIs and monosialodihexosyl GM3s. Furthermore, the levels of total GM3s were negatively correlated with sperm progressive motility and total motility. The widespread lipidomic shifts identified herein could partially explain the low sperm motility. This study presented a useful and quantitative lipidomic data repository of asthenozoospermic sperms to facilitate further mechanistic pursuits.

Spermatozoa membrane contains a heterogeneous mixture of phospholipids, glycolipids and sterols. Changes in the composition of the sperm plasma membrane phospholipids and fatty acids may culminate in membrane fluidity alteration, which may lead to impaired motility [6]. Lipidomics is a relatively new discipline that emerged in 2003. It essentially relies on the principles of analytical chemistry, which primarily centralized on mass spectrometry [20]. The fatty acid content of sperm cells has been a topic of investigation for several years [6]. Lipidomics has already been used to elucidate fatty acid groups or classes in spermatozoa from different species [22].The fatty acids lipidome of seminal fluid varied markedly between thenormozoospermia, oligoasthenoteratozoospermia, asthenozoospermia and varicocoele [16]. It has been found that the composition of saturated and unsaturated fatty acids in sperm is related to its motility, and asthenozoospermic semen samples showed lower levels of polyunsaturated fatty acids and higher levels of saturated fatty acids compared to normozoospermic samples [17]. A positive correlation between the levels of very long chain polyunsaturated fatty acids with sperm count and total motility count has been revealed by gas chromatography [38]. A significant higher cholesterol sulfate/seminolipid ratio in semen of oligoasthenozoospermic patients was observed compared with normal motility values [39].

Lipids are critical regulators of mammalian sperm function, enabling the process of capacitation, triggering acrosome exocytosis and ultimate fertilization [40]. However, the majority of published studies have investigated fatty acid composition of phospholipids, but have not achieved a satisfactory lipidomic coverage [6, 10, 38]. Since the selected reaction monitoring method is utilized in targeted lipidomics, it has provided high sensitivity for quantitative lipid analyses. With the advancement of technology, it is possible to detect and quantify low abundance lipids in human sperms with high sensitivity. In the present study, Exion UPLC coupled with a SCIEX QTRAP 6500 PLUS system was first used for the study of lipidomics in human spermatozoa. Through highcoverage targeted lipidomics, 25 individual lipid classes were detected and analyzed. Cho, PE, LPI and GM3 in asthenozoospermic spermatozoa were significantly higher those in the healthy group. It has been previously demonstrated that cholesterol content and cholesterol/ phospholipid ratio were significantly higher in asthenozoospermia and oligoasthenoteratozoospermia [12, 41]. In accordance with the previous studies, higher cholesterol content was found in asthenozoospermicspermatozoa in this study. Little is known about the relationship between sperm phospholipid composition and male infertility. This study found that the levels of total PE and LPI were significantly increased in asthenozoospermic spermatozoa compared to the healthy group. Gangliosides, including GM1, GM3 and GD1a/GDlb, constitute a large family of sialic acid-containing glycosphingolipids, which play a key regulatory role in diverse cellular processes [42]. GM1 localization is highly conserved in the spermatozoa of diverse mammalian species, and changes in GM1 localization patterns in human sperms correspond to male fertility [43]. It has been shown that ganglioside GM1 regulate sperm acrosome exocytosis (AE) and fertilization competence through CaV2.3, and its activity has been highly controversial in sperms [40]. Fertilization is characterized by the triggering of longlasting calcium $\left(\mathrm{Ca}^{2+}\right)$ oscillations in the egg cytoplasm. It was noted that intracellular $\mathrm{Ca}^{2+}$ levels were increased, accompanied with a higher concentration of GT1b in a dose-dependent manner during oocyte IVM [44]. Accumulating data also showed that gangliosides were involved in regulation of cell proliferation, and GM1 could inhibit EGFR activation and promote contact inhibition of growth through regulating the distribution of EGFR from GEM domain to caveolae domain [45]. GM3 was thought to be involved in the modulation of various biological processes including cell proliferation and differentiation, apoptosis, embryogenesis, oncogenesis, etc. $[37,46]$. Cell lines with high GM3 content showed decreased cell mobility, invasiveness and metastasis [37, 46]. GM3 could inhibit cell proliferation by perturbing the expression of factors modifying the cell cycle [37, 46]. In particular, GM3s appear to be the major ganglioside in the male reproductive system, and several studies have demonstrated their abundance in bovine, ovine, and human sperms [47-50]. GM3s are enriched in membrane rafts and localized to equatorial segment in mouse sperms [47]. GM3s are important component of lipid rafts, and their movements make sperms competent for fertilization [51]. GM3 might play a key role in 
spermatozoa maturation and fertility [52]. Although, due to technical reasons, we did not detect other gangliosides except GM3, it was found that the total GM3s levels in asthenozoospermic spermatozoa were significantly increased compared to the healthy group. Since GM3 was involved in transmembrane signaling modulation of growth factor receptor activities, as well as cell adhesion and motility, it was supposed that the increase of GM3 level might be an important reason for the decline of sperm motility in asthenozoospermic spermatozoa. Among the 479 lipid molecular species that were detected and analyzed, 48 lipid molecules were found to be significantly different between normozoospermic and asthenozoospermic spermatozoa. These 48 altered lipids belonged to 10 lipid classes, including PE, PC, PG, LPI, LPS, Cer, GM3, CL, DAG and TAG. Interestingly, it was found that among these altered lipids between the two groups, the glycerophospholipids and sphingolipids were significantly increased, but the glycolipids were significantly down-regulated in asthenozoospermic spermatozoa. These results suggested that increased phospholipids and reduced glycerolipids in spermatozoa were associated with impaired sperm motility.

The exact lipid species that correlate with sperm motility have not been conclusively established. The present study demonstrated that amongall 25 individual lipid classes that were detected and analyzed, there was a strong negative correlation between the total GM3s and LPI levels and sperm progressive motility. Among the altered molecular species between normozoospermic and asthenozoospermic spermatozoa, 10 lipids were negatively correlated with sperm progressive motility. Interestingly, five GM3 molecular species were negatively correlated with sperm progressive motility. Many studies have demonstrated that GM3s bind to the extracellular domain of epidermal growth factor receptor (EGFR), disrupting its dimerization and autophosphorylation, and inhibiting the tyrosine kinase activity of EGFR [52]. Exposure of COCs to exogenous GM3 could suppress meiotic maturation and restrict the expansion of cumulus cells during meiotic maturation via EGFR mediated PI3K/AKT signaling pathways, and could initiate apoptosis at high concentrations [53]. Epidermal growth factor (EGF) is one of the important cytokines that plays a role in spermatogenesis, spermatozoa epididymal maturation and capacitation. EGF can increase sperm concentration by stimulating the meiotic phase of spermatogenesis [54, 55], and stimulate human sperm capacitation by activating the tyrosine kinase of the EGFR [56]. EGF can also affect sperm motility parameters depending on its concentration and exposure time [57]. In asthenozoospermic spermatozoa, increased GM3s levels may inhibit the EGFR activity, which leads to decreased sperm motility. Incubation of human sperms with micelles made from glycerophospholipid mixtures increased sperm motility, which could be a relevant approach for the treatment of infertility in males [58]. It has also been found that EGF added to chilled ram sperms at concentrations of $200 \mathrm{ng} \cdot \mathrm{ml}^{-1}$ could significantly increase sperm total motility and progressive movement [57]. Herein, we hypothesized that supplementation of $200 \mathrm{ng} \cdot \mathrm{ml}^{-1} \mathrm{EGF}$ in the fertilization culture medium and then incubating the sperm at $37^{\circ} \mathrm{C}$ under $5 \% \mathrm{CO}_{2}$ might improve sperm motility and enhance reproductive effects.

It should be noted that this study had some limitations. Firstly, we used a small sample comprising of 12 asthenozoospermia and 12 normozoospermia participants to elucidate the differences in lipids composition between the normozoospermic and asthenozoospermic spermatozoa, further validation from an independent and larger cohort of patients was needed. Based on the current study design, it was thus not possible to establish a direct association between the lipidomic changes and asthenozoospermia. In addition, the dysregulation of GM3s enrichment observed in asthenozoospermic spermatozoa awaited further mechanistic validation, although our preliminary evidence suggested that the increases in GM3s might be associated with low sperm motility. Finally, in order to obtain pure sperms, we used gradient centrifugation technology that could not separate seminal plasma at the same time. Therefore, we did not compare the differences in lipids composition between the normozoospermic and asthenozoospermic seminal plasma at the same time. In the future, we needed to compare the seminal plasma lipid composition between normozoospermia and asthenozoospermia patients. This would help reveal the reasons for the elevated GM3s levels in asthenozoospermic sperms, and establish a method to rapidly evaluate sperm quality by detecting the lipids content of seminal plasma.

\section{Conclusions}

In conclusion, our work presented the first detailed overview of the lipid composition of the human sperm through high-coverage targeted lipidomics. By comparing the differences in the contents of lipids between normozoospermic and asthenozoospermic spermatozoa, we found that several unique lipid classes and lipid molecular species were linked to sperm motility, providing new possibilities for further mechanistic pursuits. To our knowledge, this was the first study to establish a correlation between the motility of human sperm and GM3 level.

\section{Abbreviations}

AR: Acrosomereaction; Cho: cholesterols; PC: phosphatidylcholines; PE: Phosphatidylethanolamines; PS: Phosphatidylserines;

PI: Phosphatidylinositols; PG: Phosphatidylglycerols; PA: Phosphatidic acids; 
LBPA: Lyso-bisphosphatidic acids; LPC: Lysophosphatidylcholine; LPI: Lysophosphatidylinositols; CL: Cardiolipins; SM: Sphingomyelins; Cer: Ceramides; GluCer: Glucosylceramides; GM3: Monosialodihexosylgangliosides; DAG: Diacylglycerol; TAG: Triacylglycerols; CE: Cholesteryl esters

\section{Supplementary Information}

The online version contains supplementary material available at https://doi. org/10.1186/s12958-021-00792-3.

\section{Additional file 1. \\ Additional file 2}

\section{Acknowledgements}

We are grateful to Guanghou Shui and Sin Man Lam of the company Lipid ALL Technologies Ltd for skillful technical assistance. We also appreciate the valuable comments from other members of our laboratory.

\section{Authors' contributions}

B.L. and J.L.H. conceived and designed the experiments. M.W. L.L. J.W. X.H.M. H.D. Z. Y.C. and B.K. provided the clinical samples. S.Q.C.,B.L. and M.W. carried out the lipidomics, analyzed the experiments and wrote the manuscript. All the authors were involved in revising the manuscript for important intellectual contents and approving the final version for publication.

\section{Funding}

This study was supported by grants from the National Natural Science Foundation of China (31801250), the Natural Science Foundation of Shaanxi Province, China (2021JM-244) and Scientific and Technical Innovatory Project of Tangdu Hospital (2017LCYJ001and 2019JSYJ003).

\section{Availability of data and materials}

The datasets used or analyzed during the current study are available from the corresponding author on reasonable request.

\section{Declarations}

\section{Ethics approval and consent to participate}

This study was approved by the Internal Review and Ethics Boards of Tangdu Hospital, and written informed consent was obtained from all controls and patients at enrollment. This study was conducted in accordance with the Declaration of Helsinki.

\section{Consent for publication}

This manuscript has not been published or presented elsewhere and is not under consideration by another journal. All study participants provided informed consent and approved it for publication.

\section{Competing interests}

The authors declare that they have no conflicts of interest.

Received: 20 December 2020 Accepted: 24 June 2021

Published online: 07 July 2021

\section{References}

1. Bolla JR, Agasid MT, Mehmood S, Robinson CV. Membrane Protein-Lipid Interactions Probed Using Mass Spectrometry. Annu Rev Biochem. 2019;88: 85-111

2. Schuhmacher $M$, Grasskamp AT, Barahtjan $P$, Wagner N, Lombardot B, Schuhmacher JS, Sala P, Lohmann A, Henry I, Shevchenko A, et al. Live-cell lipid biochemistry reveals a role of diacylglycerol side-chain composition for cellular lipid dynamics and protein affinities. Proc Natl Acad Sci U S A. 2020; 117:7729-38.

3. Grouleff J, Irudayam SJ, Skeby KK, Schiott B. The influence of cholesterol on membrane protein structure, function, and dynamics studied by molecular dynamics simulations. Biochim Biophys Acta. 2015;1848:1783-95.

4. Casares D, Escriba PV, Rossello CA. Membrane Lipid Composition: Effect on Membrane and Organelle Structure, Function and Compartmentalization and Therapeutic Avenues. Int J Mol Sci. 2019;20:2167.
5. Nielson JR, Rutter JP. Lipid-mediated signals that regulate mitochondrial biology. J Biol Chem. 2018;293:7517-21

6. Lenzi A, Picardo M, Gandini L, Dondero F. Lipids of the sperm plasma membrane: from polyunsaturated fatty acids considered as markers of sperm function to possible scavenger therapy. Hum Reprod Update 1996;2: 246-256.

7. Salvolini E, Buldreghini E, Lucarini G, Vignini A, Lenzi A, Di Primio R, Balercia $\mathrm{G}$. Involvement of sperm plasma membrane and cytoskeletal proteins in human male infertility. Fertil Steril 2013;99:697-704.

8. Lucio CF, Brito MM, Angrimani D, Belaz K, Morais D, Zampieri D, Losano J, Assumpcao M, Nichi M, Eberlin MN, Vannucchi Cl. Lipid composition of the canine sperm plasma membrane as markers of sperm motility. Reprod Domest Anim. 2017:52(Suppl 2):208-13.

9. Rivera-Egea R, Garrido N, Sota N, Meseguer M, Remohi J, Dominguez F. Sperm lipidic profiles differ significantly between ejaculates resulting in pregnancy or not following intracytoplasmic sperm injection. J Assist Reprod Genet. 2018;35:1973-85.

10. Andersen JM, Ronning PO, Herning H, Bekken SD, Haugen TB, Witczak O. Fatty acid composition of spermatozoa is associated with BMl and with semen quality. Andrology 2016;4:857-865.

11. Aksoy Y, Aksoy H, Altinkaynak K, Aydin HR, Ozkan A. Sperm fatty acid composition in subfertile men. Prostaglandins Leukot Essent Fatty Acids 2006;75:75-79.

12. Garolla A, Sabovic I, Tescari S, De Toni L, Menegazzo M, Cosci I, De Filippis $\checkmark$, Giarola M, Foresta C. Impaired sperm function in infertile men relies on the membrane sterol pattern. Andrology 2018;6:325-334.

13. Choy JT, Eisenberg ML. Male infertility as a window to health. Fertil Steril 2018, 110:810-814

14. Nowicka-Bauer K, Lepczynski A, Ozgo M, Kamieniczna M, Fraczek M, Stanski L, Olszewska M, Malcher A, Skrzypczak W, Kurpisz MK. Sperm mitochondrial dysfunction and oxidative stress as possible reasons for isolated asthenozoospermia. J Physiol Pharmacol. 2018;69:403-17.

15. Shahrokhi SZ, Salehi P, Alyasin A, Taghiyar S, Deemeh MR. Asthenozoospermia: Cellular and molecular contributing factors and treatment strategies. Andrologia 2020;52:e13463.

16. Zerbinati C, Caponecchia L, Rago R, Leoncini E, Bottaccioli AG, Ciacciarelli M, Pacelli A, Salacone P, Sebastianelli A, Pastore A, et al: Fatty acids profiling reveals potential candidate markers of semen quality. Andrology 2016:4: 1094-1101.

17. Tavilani H, Doosti M, Nourmohammadi I, Mahjub H, Vaisiraygani A, Salimi S, Hosseinipanah SM. Lipid composition of spermatozoa in normozoospermic and asthenozoospermic males. Prostaglandins Leukot Essent Fatty Acids 2007:77:45-50.

18. Wenk MR. Lipidomics: new tools and applications. Cell 2010;143:888-895.

19. Wang R, Li B, Lam SM, Shui G. Integration of lipidomics and metabolomics for in-depth understanding of cellular mechanism and disease progression. J Genet Genomics. 2020;47:69-83.

20. Lam SM, Tian H, Shui G. Lipidomics, en route to accurate quantitation. Biochim Biophys Acta Mol Cell Biol Lipids. 2017;1862:752-61.

21. Lam SM, Wang Z, Li B, Shui G. High-coverage lipidomics for functional lipid and pathway analyses. Anal Chim Acta. 2021;1147:199-210.

22. Evans HC, Dinh TTN, Hardcastle ML, Gilmore AA, Ugur MR, Hitit M, Jousan FD, Nicodemus MC, Memili E. Advancing Semen Evaluation Using Lipidomics. Front Vet Sci. 2021:8:601794.

23. Lee HC, Yokomizo T. Applications of mass spectrometry-based targeted and non-targeted lipidomics. Biochem Biophys Res Commun. 2018;504:576-81.

24. Lam SM, Tong L, Duan X, Petznick A, Wenk MR, Shui G. Extensive characterization of human tear fluid collected using different techniques unravels the presence of novel lipid amphiphiles. J Lipid Res. 2014;55:289-98,

25. Song JW, Lam SM, Fan X, Cao WJ, Wang SY, Tian H, Chua GH, Zhang C, Meng FP, Xu Z, et al. Omics-Driven Systems Interrogation of Metabolic Dysregulation in COVID-19 Pathogenesis. Cell Metab. 2020;32:188-202. e185.

26. World Medical A. World Medical Association Declaration of Helsinki: ethical principles for medical research involving human subjects. JAMA 2013;310: 2191-2194

27. Cao X, Wei R, Zhang X, Zhou J, Lou J, Cui Y. Impact of human papillomavirus infection in semen on sperm progressive motility in infertile men: a systematic review and meta-analysis. Reprod Biol Endocrinol 2020, 18:38.

28. Banyra O, Nikitin O, Ventskivska I: Acute epididymo-orchitis: relevance of local classification and partner's follow-up. Cent European J Urol 2019;72: 324-329. 
29. Pereira R, Sa R, Barros A, Sousa M. Major regulatory mechanisms involved in sperm motility. Asian J Androl. 2017;19:5-14

30. Ahammad MU, Jarrell ZR, Benson AP. Sperm Collection of Differential Quality Using Density Gradient Centrifugation. J Vis Exp. 2018;141:e58833.

31. Shui G, Stebbins JW, Lam BD, Cheong WF, Lam SM, Gregoire F, Kusonoki J, Wenk MR. Comparative plasma lipidome between human and cynomolgus monkey: are plasma polar lipids good biomarkers for diabetic monkeys? PLoS One. 2011;6:e19731.

32. Lam SM, Wang Y, Duan X, Wenk MR, Kalaria RN, Chen CP, Lai MK, Shui G. Brain lipidomes of subcortical ischemic vascular dementia and mixed dementia. Neurobiol Aging 2014;35:2369-2381.

33. Shui G, Guan XL, Low CP, Chua GH, Goh JS, Yang H, Wenk MR. Toward one step analysis of cellular lipidomes using liquid chromatography coupled with mass spectrometry: application to Saccharomyces cerevisiae and Schizosaccharomyces pombe lipidomics. Mol Biosyst 2010, 6:1008-1017.

34. Wang W, Xin J, Yang X, Lam SM, Shui G, Wang Y, Huang X: Lipid-gene regulatory network reveals coregulations of triacylglycerol with phosphatidylinositol/lysophosphatidylinositol and with hexosyl-ceramide. Biochim Biophys Acta Mol Cell Biol Lipids 2019, 1864:168-180.

35. Chicco AJ, Sparagna GC. Role of cardiolipin alterations in mitochondrial dysfunction and disease. Am J Physiol Cell Physiol. 2007;292:C33-44.

36. Julienne CM, Tardieu M, Chevalier S, Pinault M, Bougnoux P, Labarthe F, Couet C, Servais S, Dumas JF. Cardiolipin content is involved in liver mitochondrial energy wasting associated with cancer-induced cachexia without the involvement of adenine nucleotide translocase. Biochim Biophys Acta. 2014;1842:726-33.

37. Zheng C, Terreni M, Sollogoub M, Zhang Y. Ganglioside GM3 and Its Role in Cancer. Curr Med Chem. 2019;26:2933-47.

38. Craig LB, Brush RS, Sullivan MT, Zavy MT, Agbaga MP, Anderson RE. Decreased very long chain polyunsaturated fatty acids in sperm correlates with sperm quantity and quality. J Assist Reprod Genet 2019, 36:1379-1385.

39. Lopalco P, Vitale R, Cho YS, Totaro P, Corcelli A, Lobasso S. Alteration of Cholesterol Sulfate/Seminolipid Ratio in Semen Lipid Profile of Men With Oligoasthenozoospermia. Front Physiol 2019;10:1344

40. Cohen R, Buttke DE, Asano A, Mukai C, Nelson JL, Ren D, Miller RJ, CohenKutner M, Atlas D, Travis AJ. Lipid modulation of calcium flux through CaV2. 3 regulates acrosome exocytosis and fertilization. Dev Cell 2014;28:310-321.

41. Saez F, Whitfield M, Drevet JR. Impairment of sperm maturation and capacitation due to diet-dependent cholesterol overload. Andrology 2019;7: 654-661.

42. Lipina C, Hundal HS. Ganglioside GM3 as a gatekeeper of obesity-associated insulin resistance: Evidence and mechanisms. FEBS Lett 2015;589:3221-3227.

43. Cardona C, Neri QV, Simpson AJ, Moody MA, Ostermeier GC, Seaman EK, Paniza T, Rosenwaks Z, Palermo GD, Travis AJ. Localization patterns of the ganglioside GM1 in human sperm are indicative of male fertility and independent of traditional semen measures. Mol Reprod Dev. 2017:84:423-35.

44. Hwang SU, Jeon Y, Yoon JD, Cai L, Kim E, Yoo H, Kim KJ, Park KM, Jin M, Kim H, Hyun SH. Effect of ganglioside GT1b on the in vitro maturation of porcine oocytes and embryonic development. J Reprod Dev. 2015:61:549-57.

45. Zhuo D, Guan F. Ganglioside GM1 promotes contact inhibition of growth by regulating the localization of epidermal growth factor receptor from glycosphingolipid-enriched microdomain to caveolae. Cell Prolif. 2019;52: e12639.

46. Prokazova NV, Samovilova NN, Gracheva EV, Golovanova NK. Ganglioside GM3 and its biological functions. Biochemistry (Mosc) 2009;74:235-249.

47. Miranda PV, Allaire A, Sosnik J, Visconti PE. Localization of low-density detergent-resistant membrane proteins in intact and acrosome-reacted mouse sperm. Biol Reprod. 2009;80:897-904.

48. Bushway AA, Clegg ED, Keenan TW. Composition and synthesis of gangliosides in bovine testis, sperm and seminal plasma. Biol Reprod. 1977; 17:432-442

49. Gore PJ, Singh SP, Brooks DE. Composition of gangliosides from ovine testis and spermatozoa. Biochim Biophys Acta. 1986;876:36-47.

50. Mack SR, Zaneveld LJ, Peterson RN, Hunt W, Russell LD. Characterization of human sperm plasma membrane: glycolipids and polypeptides. J Exp Zool. 1987;243:339-346.

51. Watanabe H, Takeda R, Hirota K, Kondoh G. Lipid raft dynamics linked to sperm competency for fertilization in mice. Genes Cells 2017:22:493-500.

52. Kim BH, Ju WS, Kim JS, Kim SU, Park SJ, Ward SM, Lyu JH, Choo YK. Effects of Gangliosides on Spermatozoa, Oocytes, and Preimplantation Embryos. Int J Mol Sci. 2019;21:106.
53. Park HJ, Chae SK, Kim JW, Yang SG, Jung JM, Kim MJ, Wee G, Lee DS, Kim SU, Koo DB. Ganglioside GM3 induces cumulus cell apoptosis through inhibition of epidermal growth factor receptor-mediated PI3K/AKT signaling pathways during in vitro maturation of pig oocytes. Mol Reprod Dev. 2017; 84:702-11.

54. Tsutsumi O, Kurachi H, Oka T. A physiological role of epidermal growth factor in male reproductive function. Science 1986;233:975-977.

55. Abe K, Eto K, Abe S. Epidermal growth factor mediates spermatogonial proliferation in newt testis. Reprod Biol Endocrinol. 2008;6:7.

56. Furuya S, Endo Y, Oba M, Suzuki S, Nozawa S. Effect of epidermal growth factor on human sperm capacitation. Fertil Steril. 1993;60:905-910.

57. Spalekova E, Makarevich AV, Lukac N. Ram Sperm Motility Parameters under The Influence of Epidermal Growth Factor. Vet Med Int. 2011;2011:642931.

58. Ferreira G, Costa C, Bassaizteguy V, Santos M, Cardozo R, Montes J, Settineri $\mathrm{R}$, Nicolson GL. Incubation of human sperm with micelles made from glycerophospholipid mixtures increases sperm motility and resistance to oxidative stress. PLoS One 2018;13:e0197897.

\section{Publisher's Note}

Springer Nature remains neutral with regard to jurisdictional claims in published maps and institutional affiliations.

\section{Ready to submit your research? Choose BMC and benefit from:}

- fast, convenient online submission

- thorough peer review by experienced researchers in your field

- rapid publication on acceptance

- support for research data, including large and complex data types

- gold Open Access which fosters wider collaboration and increased citations

- maximum visibility for your research: over $100 \mathrm{M}$ website views per year

At BMC, research is always in progress.

Learn more biomedcentral.com/submissions 